\title{
Botulinum Toxin-A Injection in the Treatment of Spasticity in a Infantile-Onset Neurodegeneration With Brain Iron Accumulation: A Case Report
}

\author{
Hwan Kwon Do, MD, Geun Yeol Jo, MD, PhD, Jun Koo Kwon, MD, Woo Jin Kim, MD \\ Department of Physical Medicine and Rehabilitation, Inje University Haeundae Paik Hospital, \\ Inje University College of Medicine, Busan, Korea
}

\begin{abstract}
Pantothenate kinase-associated neurodegeneration (PKAN) is a neurodegenerative disorder characterized by iron accumulation in the globus pallidus (GP) of the brain (neurodegeneration with brain iron accumulation [NBIA]), which is characterized by dystonia and spasticity resulting in postural difficulties. A 33-month-old boy was admitted with a pronounced gait disturbance. Marked hypertonicity in the patient's both calf muscles was noted, resulting in waddling with repeated slip-falls. NBIA was suspected by high T2 intensity in the GP on brain MRI, then it was confirmed by detecting PANK2 mutation. Botulinum toxin-A injection was administered to both calf muscles. After 2 weeks, a decrease in spasticity and an increase in range of motion were observed, and consequently, an increase in the patient's gait stability with both heels touching the ground, enabling him to walk straight independently. A definitive treatment for NBIA has not been established, and a symptomatic therapy is currently the mainstay of treatment in this case. This is the first case report of botulinum toxin injection for treatment of gait disturbance caused by spasticity in an infantile-onset PKAN.
\end{abstract}

Keywords Neurodegeneration with brain iron accumulation (NBIA), Pantothenate kinase-associated neurodegeneration (PKAN), PANK2

\section{INTRODUCTION}

Although iron accumulation in the basal ganglia and dentate nuclei in the brain is part of the normal ageing process, intense brain iron deposition could result from the pathogenesis of common, multifactorial neurodegenerative diseases, and a rare genetic disorder [1]. There are a wide spectrum of available neurological disorders

Received April 14, 2017; Accepted August 10, 2017

Corresponding author: Woo Jin Kim

Department of Physical Medicine and Rehabilitation, Inje University Haeundae Paik Hospital, Inje University College of Medicine, 875 Haeun-daero, Haeundae-gu, Busan 48108, Korea. Tel: +82-51-797-3250, Fax: +82-51-797-0298, E-mail: cyctaz@hanmail.net

ORCID: Hwan Kwon Do (http://orcid.org/0000-0002-5862-4233); Geun Yeol Jo (http://orcid.org/0000-0002-5726-060X); Jun Koo Kwon (http://orcid. org/0000-0002-8773-1599); Woo Jin Kim (http://orcid.org/0000-0001-6172-2750).

(c) This is an open-access article distributed under the terms of the Creative Commons Attribution Non-Commercial License (http://creativecommons.org/ licenses/by-nc/4.0) which permits unrestricted noncommercial use, distribution, and reproduction in any medium, provided the original work is properly cited. Copyright (C) 2018 by Korean Academy of Rehabilitation Medicine 
in adults and children that are manifested in neurodegeneration with brain iron accumulation (NBIA) [2]. The case of childhood NBIA is typically the result of a clinically and genetically heterogeneous disorder, and $50 \%$ of childhood NBIA cases include pantothenate kinaseassociated neurodegeneration (PKAN) caused by pantothenate kinase 2 gene (PANK2) mutation with a reported prevalence of 1 to 3 per million population [3]. PKAN is characterized by iron deposition in the globus pallidus (GP) of the basal ganglia before the patients attain 10 years of age $[4,5]$. Signs of abnormal corticospinal tract function such as hypertonicity, hyperreflexia, spasticity, and plantar response are usually observed accompanied by gait or postural problems and dystonia.

A definitive treatment plan for PKAN has not been established to date, and symptomatic therapy is the mainstay of treatment at present. The application of botulinum toxin-A is widely used in selective muscle injections for muscle spasticity. The use of botulinum toxin in the treatment of PKAN has only been reported once in an adult PKAN patient. However, there is no report yet on the use of botulinum toxin injection for reducing spasticity in an infantile-onset PKAN patient. This is the first case report describing the use of botulinum toxin-A injection for treating infantile-onset PKAN with spasticity.

\section{CASE REPORT}

A 33-month-old boy was admitted to the rehabilitation department with complaints of gait disturbances. The patient had a normal birth history with history of two febrile seizures at 4 and 7 months of age. The patient had severe contraction of both calf muscles and inversion of both feet, resulting in equinovarus in both lower extremities, more evident on the left side. Ankle plantarflexor spasticity measured by the Modified Ashworth Scale (MAS), was 2 on the right and 3 on the left during knee extension. Tip-toe walking and waddling gait were observed, which was found to be aggravating on standing, and it was noted that the patient had an inability to walk in a straight line with repetitive falling due to trunk imbalance. An inability of the patient to communicate with incomprehensible verbal output was confirmed as speech and language retardation by the Sequenced Language Scale for Infants (SELSI) test, and Korean version of Bayley Scale of Infant Development second edition (BSID-II) assess- ment was revealed moderate development retardation in both cognitive and motor domains. The characteristic dystonia symptom was obscured, and accompanied by sleeping problems. An ophthalmic examination was conducted to discard any ocular problems, but it could not be completed due to the patient's poor cooperation. The biochemical assessments indicated normal levels in serum electrolyte, ammonia, amino acid, liver function test, cerebrospinal fluid examinations, and metabolic disorder studies. The brain magnetic resonance imaging (MRI) demonstrated high bilateral T2 signal intensity on the anteromedial region of both GP (Fig. 1). The NBIA was suspected and a diagnostic exome sequencing test was conducted. The NBIA type 1 (PKAN) was confirmed by detection of two mutations, p.Arg440Pro and c.524delT, in PANK2 (Table 1). A comprehensive ophthalmic examination was conducted, but it could not be completed due to patient's poor cooperation. At that time, daily rehabilitation therapy was conducted to improve the balance, postural stability, and spasticity. Because the parents of the patient were against invasive treatment at first, the baclofen $20 \mathrm{mg} /$ day was started for spasticity control and the dose was increased to $45 \mathrm{mg} /$ day after 5 days. After 4 weeks, clinical symptoms remained unchanged, and the parents were persuaded to trial of botulinum toxin injection using Botox (onabotulinumtoxinA; Allergan Inc., Irvine, CA, USA) to both calf muscles while maintaining

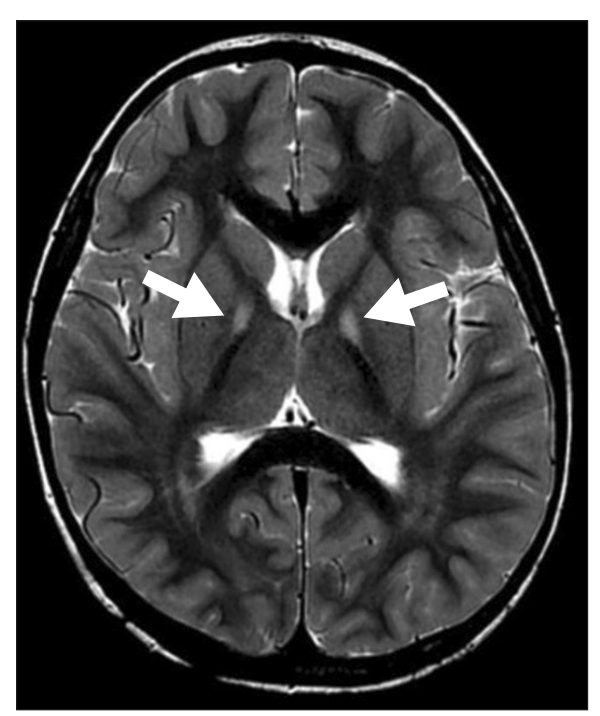

Fig. 1. T2-weighted brain magnetic resonance imaging. Arrows indicate bilateral T2 high signal intensity in anteromedial portion of both globus pallidus. 
Table 1. Type of detected gene mutation

\begin{tabular}{lllclccc}
\hline Gene & \multicolumn{1}{c}{ Mutation } & \multicolumn{1}{c}{$\begin{array}{c}\text { Mutation } \\
\text { effect }\end{array}$} & $\begin{array}{c}\text { Hetero/ } \\
\text { Homo }\end{array}$ & HGMD/OMIM & Inheritance & Classification \\
\hline PANK2 & NM_153638.2:c.1319G>C & p.Arg440Pro & Het & HARP syndrome/NBIAl & AR & Pathogenic \\
& NM_153638.2:c.524delT & p.Val175Alafs*30 & Het & HARP syndrome/NBIA1 & AR & Pathogenic \\
\hline
\end{tabular}

HGMD, Human Gene Mutation Database; OMIM, Online Mendelian Inheritance in Man; PANK2, pantothenate kinase 2; HARP, hypoprebetalipoproteinemia, acanthocytosis, retinitis pigmentosa, and pallidal degeneration; NBIA1, neurodegeneration with brain iron accumulation; AR, autosomal recessive.

Table 2. Improvement of range of motion on ankle dorsiflexion

\begin{tabular}{|c|c|c|c|c|c|c|c|c|}
\hline \multirow[b]{2}{*}{ Position } & \multicolumn{4}{|c|}{ Right } & \multicolumn{4}{|c|}{ Left } \\
\hline & $\begin{array}{c}\text { Pre- } \\
\text { injection }\left({ }^{\circ}\right)\end{array}$ & MAS & $\begin{array}{c}\text { Post- } \\
\text { injection }\left({ }^{\circ}\right)\end{array}$ & MAS & $\begin{array}{c}\text { Pre- } \\
\text { injection }\left({ }^{\circ}\right)\end{array}$ & MAS & $\begin{array}{c}\text { Post- } \\
\text { injection }\left({ }^{\circ}\right)\end{array}$ & MAS \\
\hline Knee flexion & 0 & $1+$ & 5 & 0 & -5 & 2 & 0 & 1 \\
\hline Knee extension & -20 & 2 & 0 & 0 & -30 & 3 & -10 & $1+$ \\
\hline
\end{tabular}

MAS, Modified Ashworth Scale.

the baclofen administration. The patient's weight was 14 $\mathrm{kg}$. Twenty-five units of botulinum toxin were injected into both tibialis posterior muscles to improve the ankle inversion. The plantarflexor spasticity was more severe on the left side than the right. We thus decided to inject botulinum toxin into the left plantarflexor muscles (60 units into medial gastrocnemius and 30 units into soleus) only, considering the total maximum body dose (12 units per kg total body weight) [6]. The procedure was performed under ultrasonography guidance by an experienced physiatrist. After 2 weeks, the passive range of motion (PROM) of ankle dorsiflexion during knee extension improved from $-20^{\circ}$ to $0^{\circ}$ in the right, and $-30^{\circ}$ to $-10^{\circ}$ in the left. The PROM of the ankle dorsiflexion during knee flexion, demonstrated an improvement from $0^{\circ}$ to $5^{\circ}$ in the right and from $-5^{\circ}$ to $0^{\circ}$ in the left. Further, the ankle plantarflexor spasticity reduced to MAS 0 on the right and MAS $1+$ on the left in knee extension, and to MAS 0 on the right, and MAS 1 on the left in knee flexion (Table 2). In comparison with the pre-injection state, both heels touched the ground, and the patient was able to walk independently for up to $10 \mathrm{~m}$ in a straight-line without falling or stumbling. The solid plastic ankle foot orthosis (AFO) for the patient were prescribed, and the rehabilitation therapy was continued. The patient was discharged after the 4 weeks of the botulinum toxin injection, and was consequently followed up by the out-patient department. The anti-spasticity medication was discontinued after 8 weeks of the botulinum toxin injection on the ob- serving of an improvement in ankle plantarflexor spasticity, which persisted without deterioration.

\section{DISCUSSION}

The two major phenotypes of NBIA can be classified as autosomal recessive neuroaxonal dystrophies. PKAN type 1 is caused by PANK2 mutation, causing accumulation of neurotoxin metabolite cysteine and pantotheine. The cysteine acts as a potent iron chelator and causes secondary iron accumulation, which further leads to oxidative stress and neuronal injury exacerbation [2]. The clinical manifestations of PKAN have a typically early onset in the ages of 3-4 years old children, and exhibit a classic phenotype such as pyramidal-extrapyramidal syndrome or ocular abnormalities. The characteristic symptoms are gait or postural problems, dystonia [2], speech difficulties, neuropsychiatric presentation, with variable degrees of cognitive dysfunction, sleep disorders [2] and ocular problems such as retinitis pigmentosa [7]. It is important to note that the 'Eye of the Tiger' sign in T2-weighted MRI is caused by iron accumulation in the GP, although not pathognomonic of PKAN as such MRI finding is a part of a dynamic process, and cannot be detected in the early stages of the disease [4]. The diagnosis is confirmed by identification of PNAK2 mutation in gene sequence analysis [5]. Two mutations on the PANK2 were found, pArg440Pro, which has previously been reported to be a pathogenic variant [8], combined with c524delT 
mutation, a pathogenic variant which has never been reported in previous studies and reports, and subsequently this case is the first to report it.

Curative treatment for NBIA disorders is not available to date, and the current management strategies focus on medical and surgical palliation of symptoms to improve the quality of life of the patients [3]. The characteristic effectiveness of L-dopa, iron-chelating agents, and pantothenic acid (vitamin B5) are not yet proven [2,3]. Deep brain stimulation targeting the GP as well as application of $1-\mathrm{Hz}$ repetitive transcranial magnetic stimulation on the premotor cortex could be an effective strategy in treating NBIA. Additionally, the application of trihexyphenidyl and baclofen can be used in the therapeutic management of dystonia and spasticity [2]. For now it is noted that the use of appropriate seating, orthotic devices, braces, and intervention using botulinum toxin for managing focal dystonia and/or spasticity are known to be effective.

Crisci and Esposito [9] reported that botulinum toxinA injection into the tibialis posterior and gastrocnemius muscles in a girl with PKAN with severe contraction of the calf muscle, postural instability, and gait disturbance, reduced hypertonicity and corrected internal rotation of the foot, improved standing/gait stabilities, allowing her independent walking. The use of botulinum toxin to treat other symptoms of NBIA has been scarcely described in literature. Rai et al. [10] described the dramatic effect of electromyography-guided bilateral botulinum toxin-A injection on the thyroarytenoid muscles in a 10-year-old NBIA patient with paradoxical vocal cord closing during inspiration, which were caused by adductor laryngeal breathing dystonia. In progressive disease like the NBIA, botulinum toxin-A injections are expected to control symptoms, such as hypertonicity and other life-threatening symptoms.

Controlling of the early symptoms of progressive spasticity with botulinum toxin-A injection with successive conventional rehabilitation therapy, application of AFO, and appropriate oral anti-spasticity medication, may assist in prolonging independent ambulation and hence amplify quality of life in NBIA patients. Since NBIA is a progressive disease, and the effects of the botulinum toxin are not permanent in nature, long-term observations on NBIA patients undergoing rehabilitation therapy, orthosis, and the use of anti-spasticity medications with periodic botulinum toxin injections would be required to improve patient outcomes.

\section{CONFLICT OF INTEREST}

No potential conflict of interest relevant to this article was reported.

\section{REFERENCES}

1. Gelman N, Gorell JM, Barker PB, Savage RM, Spickler EM, Windham JP, et al. MR imaging of human brain at 3.0 T: preliminary report on transverse relaxation rates and relation to estimated iron content. Radiology 1999;210:759-67.

2. Kurian MA, McNeill A, Lin JP, Maher ER. Childhood disorders of neurodegeneration with brain iron accumulation (NBIA). Dev Med Child Neurol 2011;53:394404.

3. Gregory A, Polster BJ, Hayflick SJ. Clinical and genetic delineation of neurodegeneration with brain iron accumulation. J Med Genet 2009;46:73-80.

4. Schneider SA, Hardy J, Bhatia KP. Syndromes of neurodegeneration with brain iron accumulation (NBIA): an update on clinical presentations, histological and genetic underpinnings, and treatment considerations. Mov Disord 2012;27:42-53.

5. Gregory A, Hayflick SJ. Pantothenate kinase-associated neurodegeneration. In: Adam MP, Ardinger HH, Pagon RA, Wallace SE, editors. GeneReviews. Seattle: University of Washington; 1993.

6. Graham HK, Aoki KR, Autti-Ramo I, Boyd RN, Delgado MR, Gaebler-Spira DJ, et al. Recommendations for the use of botulinum toxin type $\mathrm{A}$ in the management of cerebral palsy. Gait Posture 2000;11:67-79.

7. Egan RA, Weleber RG, Hogarth P, Gregory A, Coryell J, Westaway SK, et al. Neuro-ophthalmologic and electroretinographic findings in pantothenate kinase-associated neurodegeneration (formerly HallervordenSpatz syndrome). Am J Ophthalmol 2005;140:267-74.

8. Seo JH, Song SK, Lee PH. A novel PANK2 mutation in a patient with atypical pantothenate-kinase-associated neurodegeneration presenting with adult-onset Parkinsonism. J Clin Neurol 2009;5:192-4.

9. Crisci C, Esposito M. Efficacy of botulinum toxin A treatment in a case of pantothenate kinase associated 
neurodegeneration (PKAN). BMJ Case Rep 2011 May 16 [Epub]. http://doi.org/10.1136/bcr.07.2011.4514. 10. Rai V, Goyal V, Shukla G, Rath G, Behari M. Adductor laryngeal breathing dystonia in NBIA treated with botulinum toxin-A. Ann Indian Acad Neurol 2013;16:40910. 